\title{
NEUTROPHIL TO LYMPHOCYTE RATIO VS PLATELETS TO LYMPHOCYTE RATIO: BIOMARKERS TO PREDICT SEVERITY OF DISEASE AND THEIR COMPARISON IN PATIENTS OF COVID-19
}

\author{
Muhammad Adil, Zahid Farooq Baig*, Muhammad Amir**, Sohail Saqib Chatha, Aamir Habib***, Muhammad Majid**** \\ Combined Military Hospital Thal/National University of Medical Sciences (NUMS) Pakistan, *Combined Military Hospital Lahore/National \\ University of Medical Sciences (NUMS) Pakistan, ${ }^{* *}$ Combined Military Hospital Peshawar/National University of Medical Sciences (NUMS) \\ Pakistan, ${ }^{* * *}$ Combined Military Hospital Bannu/National University of Medical Sciences (NUMS) Pakistan, ${ }^{* * *}$ Combined Military Hospital \\ Kohat/National University of Medical Sciences (NUMS) Pakistan
}

\begin{abstract}
Objective: To determine and compare Neutrophil to Lymphocyte Ratio (NLR) and Platelet to Lymphocyte Ratio (PLR) in predicting severity of disease in patients with COVID-19.

Study Design: Cross sectional comparative study.

Place and Duration of Study: Department of Medicine, Combined Military Hospital, Thal, from Apr to Jul 2020. Methodology: Sixty One patients of COVID-19 confirmed through polymerase chain reaction were recruited and divided into severe and non severe disease. Complete blood counts were done. Neutrophil to Lymphocyte Ratio and Platelet to Lymphocyte Ratio were calculated and analyzed.

Results: Fourteen patients (23\%) of severe disease had mean age of $49.93 \pm 19.42$ and 47 patients (77\%) with nonsevere disease had mean age of $33.32 \pm 9.16$. The mean Neutrophil to Lymphocyte Ratio and Platelet to Lymphocyte ratio in patients who had severe disease was $7.20 \pm 4.20$ and $204.25 \pm 148.42$ ( $p=0.001$ and $p=0.026)$ respectively. The diagnostic performance of both Neutrophil to Lymphocyte Ratio and Platelet to Lymphocyte Ratio produced statistically significant area under the curve (AUC), $(p<0.001)$. The adjusted and unadjusted area under curve for Neutrophil to Lymphocyte Ratio was 0.92 (95\% CI: 0.85-1.00) and 0.923 (95\% CI: 0.839-1.000) and for Platelet to Lymphocyte Ratio it was 0.883 (95\% CI: 0.781-0.985) and 0.825(95\% CI: $0.707-0.943$ ) respectively.

Conclusion: Elevated Neutrophil to Lymphocyte Ratio and Platelet to Lymphocyte Ratio are independent biomarkers which predict severity of disease in COVID-19 patients with Neutrophil to Lymphocyte Ratio being better predictor in terms of diagnostic accuracy.
\end{abstract}

Keywords: Corona virus disease 2019 (COVID-19), Neutrophil to Lymphocyte ratio, Platelet to Lymphocyte ratio.

This is an Open Access article distributed under the terms of the Creative Commons Attribution License (http://creativecommons.org/licenses/by/4.0), which permits unrestricted use, distribution, and reproduction in any medium, provided the original work is properly cited.

\section{INTRODUCTION}

Corona virus is an important human pathogen which has been present since ever, causing almost $30 \%$ of all cases of community acquired respiratory tract infections during epidemics ${ }^{1}$. The name given to the virus is due to its unique crown like appearance under electron microscope. In December 2019, cluster of pneumonia patients due to a mutated and newer strain of corona virus which was named Severe Acute Respiratory Syndrome corona virus 2 (SARSCoV-2) were observed in Wuhan, Hubei province of China ${ }^{2}$. The World Health Organization has as of late formally named Novel corona pneumonia

Correspondence: Dr Muhammad Adil, Graded Medical Specialist, Combined Military Hospital, Thal Pakistan

Received: 16 Aug 2020; revised received: 17 Sep 2020; accepted: 26 Aug 2020 as Corona Virus Disease 2019 (COVID-19). This infection spread quickly from Wuhan to different countries worldwide, affecting about 10 million of world population and causing almost 500,000 deaths, causing a global health emergency ${ }^{3}$. COVID-19 spreads via direct contact and through large aerosols droplets or infected secretions thus rapidly spreading among households and in closed spaces. The disease spectrum of COVID-19 is wide, ranging from asymptomatic or mild illness in almost $80 \%$ of patients to more severe form causing respiratory failure at the other extreme within days of onset. The commonest symptoms of fever, dry cough and body aches usually occur on 4 th to 5 th day of acquiring infection. Dyspnea usually occurs on 7th to 9th day and may progress to rapid deterioration leading to Acute 
Respiratory Distress Syndrome (ARDS) and respiratory failure and therefore is an indication for hospital admission and close monitoring 4 Patients' laboratory results usually reveals diminished leukocyte and lymphocyte count, thrombocytopenia, raised transaminases, lactate dehydrogenase, creatinine kinase and myoglobin. Decreased lymphocyte count, high CRP $(\mathrm{C}$ - reactive protein), high serum ferritin, raised $\mathrm{LDH}$ and deranged liver functions are generally associated with severe disease ${ }^{5}$. Radiological findings of consolidation, bilateral chest infiltrates in mid lower zones and ground glass opacities are consistent with COVID-19 pneumonia 6 . The immunity developed after acquiring the infection is debatable so far and it is assumed to be short lived for few months which may predispose for re infections considering waning immunity ${ }^{7}$. The mortality rate of COVID-19 is $>2 \%$ worldwide and is more common in older patients with multiple co-morbid conditions ${ }^{8}$. High platelet count, total leukocyte count and high NLR are predictive of the severity of disease and high NLR is an independent risk factor for in-hospital-mortality in COVID-19 patients, which is being observed in many studies in china ${ }^{9}$. Earlier recognition of such patients warrants intensive and prompt management which can decrease morbidity and mortality.

Hematological biomarkers which can predict inflammation and immune responses have been widely studied in rheumatological and malignant diseases. These ratios are calculated from a simple blood test which is readily available even in the smaller health facilities with scarce resources, being very cost effective. These biomarkers are not being studied in COVID-19 patients of our population so we attempted to determine the efficacy of NLR and PLR as potential biomarkers for predicting severity of disease and comparing both in terms of diagnostic accuracy.

\section{METHODOLOGY}

This study was conducted at department of Medicine in Combined Military Hospital, Thal, from April 2020 to July 2020 after seeking prior approval by Ethics Review Board (Certificate Ser No.1/ERC-105341/trg-20) and informed written consent taken from all patients. Data of all patients was secured through password protected Health Management System (HMS). A total of 61 patients presenting in medical OPD of $\mathrm{CMH}$ Thal, aged between 20-85 years old, who had COVID-19 confirmed by RT-PCR (Gene-sig by primer design UK) for SARS-COV- 2 through nasopharyngeal/ oropharyngeal swabs, were recruited in this study via non probability consecutive sampling. Exclusion criteria included patients less than 18 years of age, those with prior history of asthma/COPD, Ischemic heart disease, Chronic liver or kidney disease, hematological/ autoim-mune disease or malignancy, patients on immu-nosuppressant drugs or long term steroids. History was obtained followed by clinical examination and findings were recorded. Complete baseline investigations and chest $x$-rays were done in all patients. NLR and PLR were calculated as ratios from complete blood picture (SYSMEX KX21 analyzer). Based on complete clinical data, history, symptoms/signs patients were grouped into severe and non severe categories in accordance with $\mathrm{WHO}$ guidelines ${ }^{10}$. Non-severe category patients met following criteria: (1) positive epidemiological history (2) Fever or other respiratory manifestations, (3) radiological findings consistent with viral pneumonia (4) Positive RT-PCR for SARS-CoV-2 RNA. Severe category patients also had one or more of the accompanying conditions in addition to consistent history and symptoms: (1) Shortness of breath, (2) RR $\geq 30$ times/min, (3) Oxygen saturation (Resting state) $\leq 93 \%$, (4) Multiorgan involvement.

Data was entered and analyzed in SPSS version 23.0. Continuous variables were presented in the form of Mean \pm SD and categorical variables in form of frequency and percentages. Data was grouped according to the severity of disease: severe and non-severe. Independent sample t-test was applied for the continuous variables and Fisher's exact test was applied to categorical variables. The optimal cut-off values for the NLR and PLR was obtained by ROC analysis. Binary 
logistic regression was applied to determine the influence of age and gender. The sensitivity, specificity, PPV, NPV, likelihood ratio of positive (LHR+), likelihood ratio of negative (LHR-), odds ratio adjusted, crude odds ratio, AUC (Area under curve) and adjusted AUC with 95\% confidence interval and $p$-values where needed were calculated. The $p$-value $\leq 0.05$ were considered significant.

\section{RESULTS}

In our study population, out of 61 there were $14(23 \%)$ severe cases with mean age of $49.93 \pm 19.42$ and $47(77 \%)$ of non-severe cases

Table-I: Baseline characteristics and results of neutrophil to lymphocyte ratio, platelet to lymphocyte ratio in COVID-19 patients.

\begin{tabular}{|c|c|c|c|}
\hline Characteristics & Non-Severe (47) & Severe (14) & $p$-value \\
\hline Age (years) & $33.32 \pm 9.16$ & $49.93 \pm 19.42$ & 0.007 \\
\hline \multicolumn{4}{|l|}{ Gender } \\
\hline $\begin{array}{l}\text { Male } \\
\text { Female }\end{array}$ & $\begin{array}{c}40(65.6 \%) \\
7(11.5 \%)\end{array}$ & $\begin{array}{c}13(21.3 \%) \\
1(1.6 \%)\end{array}$ & 0.668 \\
\hline Total Leucocyte Count (x $10^{9}$ cells / L) & $7.857 \pm 2.93$ & $11.56 \pm 5.14$ & 0.021 \\
\hline (Neutrophills (x $10^{9}$ cells / L) & $4.94 \pm 2.74$ & $9.46 \pm 4.86$ & 0.004 \\
\hline Lymphocytes (x $10^{9}$ cells / L) & $2.31 \pm 0.65$ & $1.50 \pm 0.59$ & 0.0001 \\
\hline Platelets ( $\times 10^{9}$ cells / L) & $219.06 \pm 92.78$ & $264.71 \pm 146.05$ & 0.166 \\
\hline Neutrophil to Lymphocyte Ratio (NLR) & $2.36 \pm 1.90$ & $7.20 \pm 4.20$ & 0.001 \\
\hline Platelet to Lymphocyte (PLR) & $103.10 \pm 57.74$ & $204.25 \pm 148.42$ & 0.026 \\
\hline
\end{tabular}

Table-II: Sensitivity, specificity, predictive and likelihood ratios in COVID-19 patients.

\begin{tabular}{|c|c|c|c|c|}
\hline \multirow{2}{*}{ Measures } & \multicolumn{2}{|c|}{$\begin{array}{l}\text { Neutrophil to Lymphocyte Ratio } \\
\text { (NLR) }\end{array}$} & \multicolumn{2}{|c|}{ Platelet to Lymphocyte (PLR) } \\
\hline & Value & $\begin{array}{l}\text { Confidence } \\
\text { Interval }\end{array}$ & Value & $\begin{array}{l}\text { Confidence } \\
\text { Interval }\end{array}$ \\
\hline Cut-Off & 2.4 & & 120 & \\
\hline Sensitivity & 92.86 & $66.13-99.82$ & 64.29 & $35.14-87.24$ \\
\hline Specificity & 68.09 & $52.88-80.91$ & 74.47 & $59.65-86.06$ \\
\hline Positive Likelihood Ratio (+LHR) & 2.91 & $1.87-4.53$ & 2.52 & $1.35-4.70$ \\
\hline Negative Likelihood Ratio (-LHR) & 0.10 & $0.02-0.70$ & 0.48 & $0.23-0.99$ \\
\hline Positive Predictive Value (PPV) & 46.43 & $35.77-57.42$ & 42.86 & $28.64-58.36$ \\
\hline Negative Predictive Value (NPV) & 96.97 & $82.73-99.53$ & 87.50 & $77.27-93.51$ \\
\hline Accuracy & 73.77 & $60.93-84.20$ & 72.13 & $59.17-82.85$ \\
\hline Cut-Off & 3.0 & - & 150 & - \\
\hline Sensitivity & 92.86 & $66.13-99.82$ & 64.29 & $35.14-87.24$ \\
\hline Specificity & 82.98 & 69.19-92.35 & 91.49 & $76.62-97.63$ \\
\hline Positive Likelihood Ratio (+LHR) & 5.46 & $2.85-10.43$ & 7.55 & $2.74-20.85$ \\
\hline Negative Likelihood Ratio (-LHR) & 0.09 & $0.01-0.57$ & 0.39 & $0.19-0.79$ \\
\hline Positive Predictive Value (PPV) & 61.90 & $45.95-75.64$ & 69.23 & $44.91-86.13$ \\
\hline Negative Predictive Value (NPV) & 97.50 & $85.45-99.62$ & 89.58 & $80.90-94.58$ \\
\hline Accuracy & 85.25 & $73.83-93.02$ & 85.25 & $73.83-93.02$ \\
\hline Cut-Off & 3.3 & - & 180 & - \\
\hline Sensitivity & 85.71 & $57.19-98.22$ & 35.71 & $12.76-64.86$ \\
\hline Specificity & 89.36 & $76.90-96.45$ & 95.74 & $85.46-99.48$ \\
\hline Positive Likelihood Ratio (+LHR) & 8.06 & $3.42-18.96$ & 8.39 & $1.83-38.66$ \\
\hline Negative Likelihood Ratio (-LHR) & 0.16 & $0.04-0.58$ & 0.67 & $0.45-1.00$ \\
\hline Positive Predictive Value (PPV) & 70.59 & $50.49-84.96$ & 71.43 & $35.18-92.01$ \\
\hline Negative Predictive Value (NPV) & 95.45 & $65.29-98.70$ & 83.33 & $77.11-88.13$ \\
\hline Accuracy & 88.52 & $77.78-95.26$ & 81.97 & $70.02-90.64$ \\
\hline
\end{tabular}


with the mean age of $33.32 \pm 9.16$. In severe cases, average age was significantly higher than the non-severe cases $(p=0.007)$. The overall distribution of the male and female in present study was $53(86.9 \%)$ and $8(13.1 \%)$ respectively and no gender based significant difference was noted $(p=0.668)$ in terms of severity of disease. The mean NLR and PLR of patients with severe disease were significantly higher than those of patients with non severe disease $(p=0.001$ and $p=0.026$, respectively) (table-I).

The optimal cut-off values for univariate and multivariate analysis are presented in fig- 1 and the distribution of NLR and PLR in severe and non-severe patients is presented in fig- 2 and fig- 3 respectively. The diagnostic performance of both NLR and PLR after adjustment for age and gender produced statistically significant areas under the curve $(p<0.001)$. The adjusted and unadjusted AUC for the NLR was 0.92 (95\% CI: 0.85-1.00) and 0.923 (95\% CI: 0.839-1.000) respectively. The adjusted and unadjusted AUC for the PLR was 0.883 (95\% CI: 0.781-0.985) and 0.825 (95\% CI: $0.707-0.943)$ respectively. The logistic regression analysis was applied to identify the factors like age or gender that may affect COVID-19 progression. The odds ratio (ORa) was calculated by multivariate analysis. Increased age showed a significant association with the severity of disease however gender did not add a significant part in both prediction models. A positive association of NLR was found in determining the risk of severe disease. PLR also showed positive association but when diagnostics accuracy tests were performed PLR was found to be less efficient in prediction of severity of the disease as compared to the NLR.

We tested our data against three possible optimal cut-off values for both the NLR and PLR. The optimal cut-off values were 2.4, 3.0 and 3.3 for NLR. Diagnostic measures for these cut-off points are presented in table-II, the highest sensitivity was achieved at cutoff 2.4 and 3.0 whereas highest specificity was obtained at cut point 3.3. However the cutoff point 3.3 was found to be the most appropriate in terms of diagnostic measures with the sensitivity, specificity and accuracy of
$85.7 \%, 89.36 \%, 88.52 \%$. For the NLR $>3.3$ the probability for the prediction of disease severity was $70.59 \%$ (95\% CI: $50.49 \%-84.96 \%$ ). For the NLR

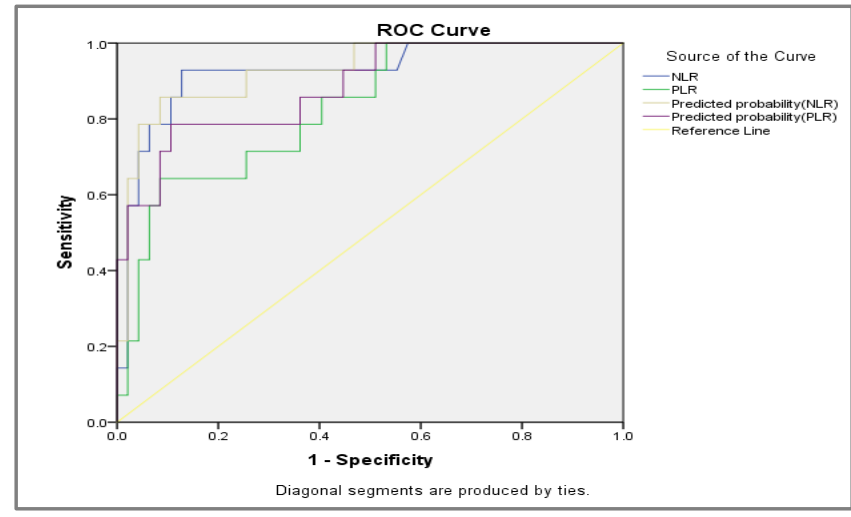

Figure-1: ROC curve to distinguish patients with severe and non-severe COVID-19.

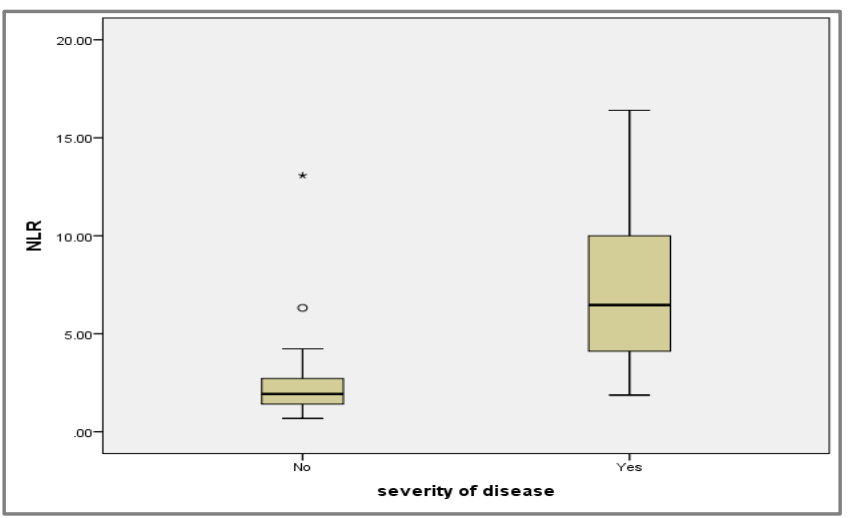

Figure-2: Distribution of neutrophil to lymphocyte ratio (NLR) in severe and non-severe COVID-19 patients.

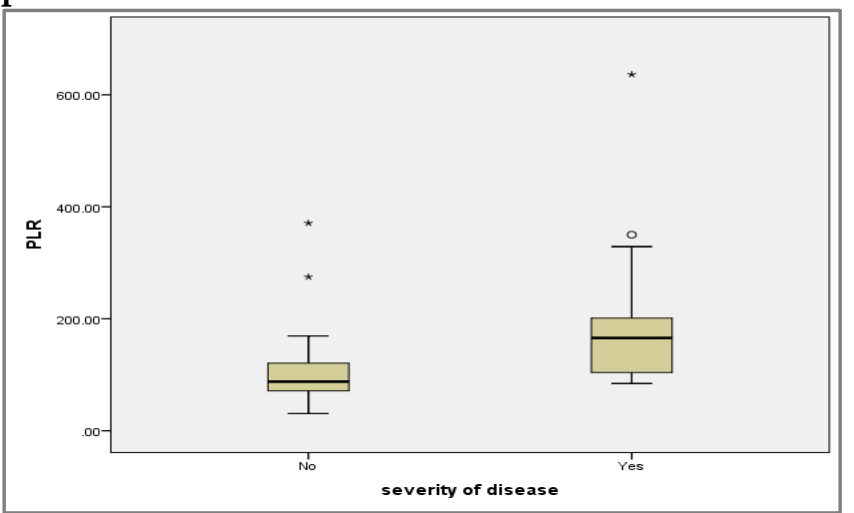

Figure-3: Distribution of platelet to lymphocyte ratio (PLR) in severe and non-severe COVID-19 patients.

$\leq 3.3$ the probability of being non-severe patient was $95.45 \%$ (65.29\%-98.70\%). Positive Likelihood ratio also showed high chances of being severe patient for NLR>3.3. The possible optimal cut-off 
values for PLR were 120, 150 and 180 . The highest sensitivity was achieved at cutoff 120 and 150 whereas highest specificity was obtained at cut point 180 . The cutoff value of 150 was found to be the most appropriate in terms of diagnostic measures with the sensitivity, specificity and accuracy of $64.29 \%, 91.49 \%$ and $85.25 \%$. For PLR $>150$, the probability for the prediction of disease severity was $69.23 \%$ (95\% CI: $44.91 \%-86.13 \%$ ). For the PLR $\leq 150$ the probability of being non-severe patient was $89.58 \%$ (95\% CI: $80.90 \%-94.58 \%$ ). Positive Likelihood ratio also showed high chances of being severe patient for PLR >150.

\section{DISCUSSION}

COVID-19, being a highly infectious and contagious disease has taken the world by storm, affecting a large population globally. It has affected global health, social life, psychological/mental health, international economy and its long term effects are yet to be accurately ascertained 11,12 . The cytokine storm syndrome associated with COVID-19 is a warning for the clinicians that impending complications are inevitable until the patient is aggressively managed in intensive care facility ${ }^{13}$.

Neutrophils, Lymphocyte and platelets are common inflammatory markers used to predict the disease progression in various diseases because of their multifaceted response during inflammation. NLR and PLR are considered better markers being ratios involving two different inflammatory biomarkers. A recent meta-analysis of 18 studies was done in china by Henry et al, including almost 3000 patients and found high TLC count, higher neutrophils, decreased lymphocyte and low platelet counts in severe patients who had ARDS secondary to COVID-19 or those who required intensive care treatment. These findings were consistent with our study except for platelet counts which was $264.71 \pm 146.05$ in patients with severe disease as compared to $219.06 \pm 92.78$ in patients with non severe disease, though the results were statistically insignificant $(p=0.166)^{14}$. Similarly, NLR and PLR are being studied in viral and bacterial pneumonias in past.
NLR was found to be more sensitive indicator of severity than neutrophils and lymphocyte individually and PLR was better predictor of mortality in bacterial pneumonias ${ }^{15,16}$. In a study by Long et al published in European journal of Respiratory Medicine and another study by Jin et al conducted in china, assessing the short term disease outcomes in COVID-19, patients who had disease progression during hospitalization had elevated mean NLR (hazard risk (HR): 2.46, 95\% CI: 1.984.57 ) and mortality rate was higher in men as compared to women (70.3 vs. $29.7 \%, p=0.016$ ). Male sex, Elevated Mean NLR and co-morbid conditions were identified as independent factors for poor clinical outcomes ${ }^{17,18}$. Similarly in a meta analysis by Perez et al, which examined mortality rate in COVID-19 patients based on gender from cases in European countries, male gender was identified as a risk factor and its association with more severe disease as compared to the female gender with male-female mortality risk ratio of 1.60 (95\% CI 1.531 .68$)^{19}$. Our study did not find any predilection of severe disease for any specific gender $(p=0.668)$ however, increasing age was found to be significantly associated with severe disease $49.93 \pm 19.42$ vs. $33.32 \pm 9.16$ years in patients with non severe disease $(p=0.007)$.

A meta-analysis of six studies and 828 patients by Lagunas et al also concluded that the patients who had high NLR and low lymphocyteto-C-reactive protein ratio had poor prognosis in COVID-19 patients ${ }^{20}$. These findings are also reflected in this study as NLR and PLR were both high in severe patients and NLR was more sensitive in predicting severity as compared to PLR ( $p=0.001$ vs. 0.026$)$ respectively. The diagnostic performance of both the NLR and PLR after adjustment for age and gender produced statistically significant areas under the curve, AUC $(p<0.001)$. The arbitrary cut off points for NLR and PLR are misleading as these values being derived from hematological parameters, which depends upon age, environment and many clinical conditions ${ }^{21}$.

In a study by yang et al, NLR AND PLR were studied in 93 laboratory confirmed patients of 
COVID-19 which revealed higher AUC for NLR as compared to PLR (0.841 vs. 0.784) and also found NLR to be positively correlated with risk of COVID-19 pneumonia while the risk associated with PLR was unclear ( $p=0.019$ vs. 0.252 ). Cut off value of 3.3 for NLR had the highest sensitivity and specificity of (0.636 and 0.88$)$ and for PLR cut off of 180 was found to have highest sensitivity and specificity (0.44 and 0.77$)$ respectively22. Our study also found close results and found NLR to be more closely related in predicting severity of disease in COVID-19 patients as compared to PLR ( $p=0.001$ vs. 0.026). Cut off value of 3.3 for NLR and 150 for PLR resulted in highest sensitivity and specificity $(0.928$ and 0.829 vs. 0.642 and 0.914 ) respectively and larger area under the curve with superior diagnostic possibility for symptoms to progress from mild to severe.

\section{LIMITATIONS OF STUDY}

There were certain limitations to this study as it was conducted in a single health facility on a population which shared similar environmental conditions and who shared common social and cultural life. A wider group of population with different environmental exposures might have added to the validity of this study. Considering the very peripheral location of this hospital with limited resources, reliability on these simple laboratory parameters proved clinically useful.

\section{ACKNOWLEDGEMENT}

We are thankful to all the authors for their valuable input and all patients who became part of this study which helped us in understanding the clinical outcomes and associated biomarkers. We are also thankful to the consultant Pathologist, Dr. Akhtar Ali and Dr. Umer Naeem, CMH Thal who verified all the laboratory results and helped us in completing this project.

\section{CONCLUSION}

Elevated NLR and PLR are independent biomarkers which predict severity of disease in COVID-19 patients. On comparison between NLR and PLR, NLR was found to be a better predictor in terms of diagnostic accuracy. Increasing age irrespective of gender was also associated with severe disease.

\section{CONFLICT OF INTEREST}

This study has no conflict of interest to be declared by any author.

\section{REFERENCES}

1. Mcintosh K. Coronavirus disease 2019 (COVID-19): Epidemiology, virology, and prevention. In: Bloom A, ed. Up-to-Date [database on the internet]. Waltham (MA): UpToDate; 2019. Available from: http://www.uptodate.com

2. Huang C, Wang Y, Li X, Ren L, Zhao J, Hu Y, et al. Clinical features of patients infected with 2019 novel coronavirus in Wuhan, China. Lancet 2020; 395(10223): 497-506.

3. World Health Organization. Director-General's opening remarks at the media briefing on COVID-19. Available from: https:// www.who.int/emergencies/diseases/novel-coronavirus-2019

4. Peiris JS, Chu CM, Cheng VC, Chan KS, Hung IF, Poon LL, et al. Clinical progression and viral load in a community outbreak of coronavirus-associated SARS pneumonia: a prospective study. Lancet 2003; 361(9371): 1767-72.

5. Terpos E, Ntanasis SI, Elalamy I, Kastritis E, Sergentanis TN, Politou $\mathrm{M}$, et al. Hematological findings and complications of COVID-19. Am J Hematol 2020; 95(7): 834-47.

6. Wong HY, Lam HY, Fong AH, Leung ST, Chin TW, Lo CS, et al. Frequency and distribution of chest radiographic findings in COVID-19 positive patients. Radiol 2020; 292(2): 72-78.

7. Nikolich ZJ, Knox KS, Rios CT, Natt B, Bhattacharya D, Fain MJ. SARS-CoV-2 and COVID-19 in older adults: what we may expect regarding pathogenesis, immune responses, and outcomes. Geroscience 2020; 42(2): 505-14.

8. Zhou F, Yu T, Du R, Fan G, Liu Y, Liu Z, et al. Clinical course and risk factors for mortality of adult inpatients with COVID-19 in Wuhan, China: a retrospective cohort study. Lancet 2020; 395(10229): 1054-62.

9. Liu Y, Du X, Chen J, Jin Y, Peng L, Wang HH, et al. Neutrophilto-lymphocyte ratio as an independent risk factor for mortality in hospitalized patients with COVID-19. J Infect 2020; 81(1): 6-12.

10. World Health Organization (WHO). Clinical management of severe acute respiratory infection when Middle East respiratory syndrome coronavirus ( ERS-CoVinfection is suspected: interim guidance. World Health Organization; 2019.

11. Cao W, Fang Z, Hou G, Han M, Xu X, Dong J, et al. The psychological impact of the COVID-19 epidemic on college students in China. Psychiatry Res 2020; 287(1):1129-34.

12. McKibbin W, Fernando R. Economics in the Time of COVID-19. London: Centre for Economic Policy Research; 2020. Chapter 3: The economic impact of COVID-19; p.45-51.

13. Henderson LA, Canna SW, Schulert GS, Volpi S, Lee PY, Kernan $\mathrm{KF}$, et al. On the alert for cytokine storm: Immunopathology in COVID-19. Arthritis Rheumatol 2020; 72(7): 1059-63.

14. Henry BM, De Oliveira MH, Benoit S, Plebani M, Lippi G. Hematologic, biochemical and immune biomarker abnormalities associated with severe illness and mortality in corona virus disease 2019 (COVID-19): a meta-analysis. Clin Chem Lab Med 2020; 58(7): 1021-28.

15. Han Q, Wen X, Wang L, Han X, Shen Y, Cao J, et al. Role of hematological parameters in the diagnosis of influenza virus infection in patients with respiratory tract infection symptoms. J Clin Lab Anal 2020; 34(5): 23191. 
16. Lee JH, Song S, Yoon SY, Lim CS. Neutrophil to lymp-hocyte ratio and platelet to lymphocyte ratio as diagnostic markers for pneumonia severity. Br J Biomed Sci 2016; 73(3): 140-42.

17. Long L, Zeng X, Zhang X, Xiao W, Guo E, Zhan W, et al. Shortterm outcomes of COVID-19 and risk factors for progression. Eur Respir J 2020; 55(5): 2000990.

18. Jin JM, Bai P, He W, Wu F, Liu XF, Han DM, et al. Gender differences in patients with COVID-19: Focus on severity and mortality. Frontiers Pub Health 2020; 8(1): 152.

19. Pérez-López FR, Tajada M, Savirón-Cornudella R, SánchezPrieto M, Chedraui P, Terán E. Coronavirus disease 2019 and gender-related mortality in European countries: A meta-analy- sis. Maturitas 2020; 141(1): 159-62.

20. Lagunas-Rangel FA. Neutrophil to lymphocyte ratio and lymphocyte to C-reactive protein ratio in patients with severe coronavirus disease 2019 (COVID-19): A meta analysis. J Med Virol 2020; 10(1): 1002.

21. Alexander N. Reference values of neutrophil-lymphocyte ratio, platelet-lymphocyte ratio and mean platelet volume in healthy adults in north central Nigeria. J Blood Lymph 2016; 6(1): 143-48.

22. Yang AP, Liu J, Tao $\mathrm{W}, \mathrm{Li}$ HM. The diagnostic and predictive role of NLR, d-NLR and PLR in COVID-19 patients. Int immunopharmacol 2020; 84(1): 106504. 